\section{Mathematical explanation}

Introduction to Mathematical Biology. By S. I. Rubinow. Pp. xiiit 386. (Wiley-Interscience: New York and London, December 1975.) \$27.00; $£ 13.55$.

THIS book is based on a course designed to develop in graduate biology students at Cornell University an awareness of and familiarity with some mathematical techniques and methods applied to biology. Apart from brief mention of the application of the least squares method to linear regression and the extraction of the parameters of multi-exponential processes by the 'peeling' method, statistical and numerical analytical techniques for processing experimental data are not featured. The author clearly believes, however, that mathematical biology should be firmly based on experimental observation. Consequently, no attempt is made to describe methods involving analogue or digital computers for handling speculative mathematical models of biological systems.

The book deals with the mathe matical explanation of five selected biological phenomena in some depth: cell growth, enzyme kinetics, tracers in physiological systems, biological fluid dynamics and diffusion in biology. The style is economical so that, for example, steady-state and presteady-state kinetics, cooperativity and allostery and the effects of inhibitors and modifiers are treated in 53 pages in the chapter on enzyme kinetics.

Where possible, the author applies fundamental concepts to a variety of biological problems. For example, an explanation of Fick's laws of diffusion leads to consideration of topics as diverse as the relationship between diffusion constant and molecular weight, pheromone interaction between animals, membrane transport, diffusion through a slab, axonic ion flow, ultracentrifugation and diffusion across capillary walls. Three other features of the book deserve mention. When a new term is introduced and defined, it is printed in boldfaced type which will facilitate revision by students. Each chapter ends with a number of problems of graded mathematical difficulty. The solutions with full explanations are given towards the end of the book.

Finally, although the author states that this is not a textbook in mathematics, there are two useful mathematical appendices for readers who have forgotten basic techniques such as the handling of matrices and determinants. Unfortunately, stu-

\section{PMO theory}

The PMO Theory of Organic Chemistry. By Michael J. S. Dewar and Ralph C. Dougherty. Pp. xiii +576. (Plenum: New York, 1975.) \$14.50.

IN the preface, the authors disclaim any intention of rewriting the whole of organic chemistry in terms of perturbational molecular orbital (PMO) theory. The text illustrates principles of such widespread application that it seems that only time has prevented them from so doing. The first three chapters introduce the theory of the method in a most readable manner. The principles are well presented, and the material could stand up in its own right as a separate text. The bulk of the text comprises three chapters with far-reaching titles -Chemical Equilibria (ch.4), Chemi-

dents of the biological sciences who could and should read this book are likely to find the price a deterrent. It deserves a place in science libraries and hopefully will be well thumbed with use by students and lecturers.

D. T. Elmore

\section{Infrared astronomy}

Infrared: The New Astronomy. By David A. Allen. Pp. 228. (Keith Reid: Sheldon, Devon, November 1975.) $£ 6.50$.

THIs is an unusual book, a semipopular one written completely from the viewpoint of the observational astronomer. It charts the rise of a new field, infrared astronomy, with which the author has been actively involved for a decade. After introductory chapters outlining a few background ideas, the techniques of observation (formidable if the only dewar you have ever handled is a thermos flask), the main observing sites, and the early history, the book discusses the different types of source seen in the infrared: solar system objects, stars and protostars, and galaxies and quasars. All are discussed on the basis of whether they are detectable in the infrared, and more particularly whether they are unusually strong emitters there. This seems to be the weakness of the observer's viewpoint, especially when he is specialising in a fairly narrow range of wavelengths (most of the book concerns the range $1-20 \mu \mathrm{m})$. No theoretical framework is provided which explains why different types of star or galaxy are of interest. So this is a book which requires a rather extensive knowledge cal Reactivity (ch.5) and Light Absorption and Photochemistry (ch.6). The final chapter is concerned with transient ions, and seems too specialised for the general audience at whom the work as a whole is aimed. Inevitably some of the material will provoke controversy, and there is a rather cavalier treatment of alternative approaches to some of the problems. For example, the summary dismissal of the Hoffmann-Woodward approach to Pericyclic Reactions, and the absence of any reference to $R$. W. Taft in the section dealing with substituent effects would be remarkable if it were not so predictable. The book succeeds admirably in its stated aim of demonstrating the power of the PMO method, and does so in such a readable manner that it will be appreciated by a wide audience.

L. Phillips

of astronomy to appreciate its qualities.

The chapters on the different types of star which radiate in the infrared, either because they are cool or more often because they are partially shrouded in dust, are outstanding, and constitute a valuable review of, and introduction to, the field.

That on galaxies and quasars, on the other hand, opens weakly: "A galaxy is some breccia constructed of all the types of object we have encountered in the last four chapters". No real effort is made to distinguish between different types of galaxy. Under "Compact Galaxies" we find I $\mathrm{Zw} 1727+50$, which is also a $\mathrm{BL}$ Lac-type object: the "Markarian galaxies" mentioned are also, in fact, Seyferts. And the chapter ends with a decidedly casual (and even for June 1974, inaccurate) account of the microwave background.

If $\mathrm{Dr}$ Allen were writing the book today, I think he might devote a larger fraction to wavelengths between $30 \mu \mathrm{m}$ and $1 \mathrm{~mm}$, where many of his sources emit most of their energy. He might also devote more space to the clouds of dust and molecules out of which form the young stars on which he is so expert.

This is a book for the professional astronomer (for whom an excellent feature will be the comprehensive list of references) or the knowledgeable amateur. How the style appeals to you will depend on your taste for extravagant metaphor. For example, synchrotron radiation is explained as "those piercing shrieks emitted by high energy electrons when they embark on their helter-skelter paths in magnetic fields".

M. Rowan-Robinson 